\title{
Direct numerical simulation of premixed flame boundary layer flashback in turbulent channel flow
}

\author{
A. Gruber ${ }^{1} \dagger$, J. H. Chen ${ }^{2}$, D. Valiev ${ }^{2,3}$ and C. K. Law ${ }^{3}$ \\ ${ }^{1}$ SINTEF Energy Research, 7465 Trondheim, Norway \\ 2 Combustion Research Facility, Sandia National Laboratories, Livermore, CA 94550, USA \\ ${ }^{3}$ Department of Mechanical and Aerospace Engineering, Princeton University, Princeton, NJ 08544, USA
}

(Received 21 December 2011; revised 22 March 2012; accepted 2 July 2012; first published online 29 August 2012)

Direct numerical simulations are performed to investigate the transient upstream propagation (flashback) of premixed hydrogen-air flames in the boundary layer of a fully developed turbulent channel flow. Results show that the well-known near-wall velocity fluctuations pattern found in turbulent boundary layers triggers wrinkling of the initially flat flame sheet as it starts propagating against the main flow direction, and that the structure of the characteristic streaks of the turbulent boundary layer ultimately has an important impact on the resulting flame shape and on its propagation mechanism. It is observed that the leading edges of the upstream-propagating premixed flame are always located in the near-wall region of the channel and assume the shape of several smooth, curved bulges propagating upstream side by side in the spanwise direction and convex towards the reactant side of the flame. These leading-edge flame bulges are separated by thin regions of spiky flame cusps pointing towards the product side at the trailing edges of the flame. Analysis of the instantaneous velocity fields clearly reveals the existence, on the reactant side of the flame sheet, of backflow pockets that extend well above the wall-quenching distance. There is a strong correspondence between each of the backflow pockets and a leading edge convex flame bulge. Likewise, high-speed streaks of fast flowing fluid are found to be always colocated with the spiky flame cusps pointing towards the product side of the flame. It is suggested that the origin of the formation of the backflow pockets, along with the subsequent mutual feedback mechanism, is due to the interaction of the approaching streaky turbulent flow pattern with the Darrieus-Landau hydrodynamic instability and pressure fluctuations triggered by the flame sheet. Moreover, the presence of the backflow pockets, coupled with the associated hydrodynamic instability and pressure-flow field interaction, greatly facilitate flame propagation in turbulent boundary layers and ultimately results in high flashback velocities that increase proportionately with pressure.

Key words: channel flow, flames, turbulent reacting flows 


\section{Introduction}

\subsection{Background}

In precombustion $\mathrm{CO}_{2}$ separation schemes hydrogen is considered as a candidate fuel for clean and efficient large-scale power generation with carbon capture and storage (CCS) where the original fuel is either reformed (natural gas) or gasified (coal) to a synthetic fuel mixture as discussed by Bolland \& Undrum (2003). The overall performance of this particular concept depends on the gas turbine hardware that can safely, economically and cleanly burn fuels that are very rich in hydrogen (up to $90 \%$ by volume). Diffusion burners are presently used for combusting hydrogen-rich fuels, but these require large amounts of dilution $\left(\mathrm{N}_{2}\right.$ and/or steam concentration typically larger than $50 \%$ by volume) in order to comply with emissions regulations. A further reduction of emissions, without increase in the energy penalty intrinsic to dilution, can only be achieved with lean-premixed (LPM) combustion. However, such burner technology for hydrogen-rich fuels does not currently operate in gas turbines (Carroni 2006). In comparison to natural gas that is normally burned in gas turbines, hydrogen is characterized by significantly higher stoichiometric combustion temperatures and much smaller volumetric calorific values. These features, coupled with the drastically reduced auto-ignition delay times, require that combustor design for hydrogen-rich fuels must be modified to account for the large increase in volumetric fuel flow rates, to avoid pre-ignition and to ensure that flashback into the premixer section of the burner does not occur.

Among the many challenges in enabling LPM hydrogen-fired gas turbines (Chiesa, Lozza \& Mazzocchi 2005), two topics stand out as major impediments to our fundamental understanding of the physical processes involved:

(a) flame flashback;

(b) flame holding and stabilization.

The latter issue is being addressed by the present authors by performing detailed direct numerical simulations (DNSs) of canonical fuel injection configurations (jet in crossflow) and focusing on the mechanism of aerodynamic stabilization of non-premixed flames in the near field of fuel injection nozzles. The flame holding behaviour is investigated as a function of injection geometrical configuration, fuel composition and injection angle (Grout et al. 2011, 2012; Kolla et al. 2012). The former topic, focusing on the characteristics of the upstream propagation of premixed, preheated hydrogen $\left(\mathrm{H}_{2}\right)$-air flames in turbulent boundary layers, against the bulk flow direction, constitutes the main concern of the present DNSs and of a parallel experimental effort (Eichler, Baumgartner \& Sattelmayer 2011; Eichler \& Sattelmayer 2011, 2012) conducted in the framework of the BIGCO2 Project and of the BIGCCS International CCS Research Center (more information is available at http://www.bigccs.no).

\subsection{Motivation}

In the context of gas turbine combustor design it is of prime importance to achieve improvements in the predictive capabilities regarding boundary layer flashback, see Fritz, Kröner \& Sattelmayer (2004) for a classification of different canonical flashback mechanisms. While boundary layer flashback has previously represented a minor issue for natural gas fired gas turbines, mounting evidence involving premixed combustion of hydrogen-rich syngas at gas turbine conditions (high pressure, high reactants' temperature) indicates that boundary layer flashback may constitute a key challenge (Mayer et al. 2011) even in the presence of large quantities of diluent (Carroni 2006). 
In fact, the increased reactivity of hydrogen-rich syngas complicates the problem of boundary layer flashback considerably. Wall-quenching studies involving $\mathrm{H}_{2}$-air premixed laminar (Dabireau et al. 2003) and turbulent (Gruber et al. 2010) flames have shown that, even if they are characterized by similar qualitative quenching behaviour as observed earlier in hydrocarbon-air flames, they exhibit quantitatively different quenching parameters: quenching Péclet number $P e_{Q}$ (quenching distance $y_{Q}$ normalized by the laminar flame thickness at quenching $\delta_{Q}$ ) and non-dimensional wall heat flux $F_{Q}$ (wall heat flux at quenching $\Phi_{w, Q}$ normalized by the undisturbed laminar flame power $\left.P_{Q}\right)$. Specifically, compared with hydrocarbon-air flames, $\mathrm{H}_{2}$-air premixed flames are able to propagate three times (in relation to the flame thickness) closer to the wall before the heat loss to the solid surface leads to quenching. This, in turn, implies that, compared with their methane-air counterparts, $\mathrm{H}_{2}$-air flames can propagate closer to the wall in regions of the boundary layer characterized by very low flow velocities; this also leads to increased heat transfer and (potential) damage to the premixer liner wall. Finally, and most importantly, using primary combustion air to flush the premixer and combustor walls, a common practice traditionally used to prevent boundary layer flashback of hydrocarbon flames, is no longer feasible when operating the gas turbine with hydrogen-rich fuels as all of the available air is needed for dilution of the highly reactive fuel.

\subsection{Previous work}

The first study to systematically investigate flashback limits is that of Lewis \& von Elbe (1943), whose model has remained as the state of the art for order-of-magnitude flashback predictions. This model, however, is based on the balance (or imbalance) between the laminar flame speed $S_{l}$ and the undisturbed boundary layer velocity at a distance from the wall corresponding to the quenching distance $u\left(y_{Q}\right)$. Consequently, intrinsic to this model is the assumption that the flame has negligible effect on the flow field. The present study shows that this assumption is not valid.

One-dimensional numerical simulations of laminar flame-wall interaction (FWI) configurations are reported in quenching studies by Hocks, Peters \& Adomeit (1981), Westbrook, Adamczyk \& Lavoie (1981), Ezekoye, Greif \& Sawyer (1992), Popp, Smooke \& Baum (1996), Egolfopoulos, Zhang \& Zhang (1997), Popp \& Baum (1997) and Dabireau et al. (2003). While boundary layer flashback is a particular type of FWI process in which the flame propagates parallel to the solid surface, the focus of these FWI studies is mainly on premixed laminar flames propagating perpendicular to the wall and stagnating on it. The configuration is also known as head-on quenching (HOQ) and, although such one-dimensional FWI studies do not directly provide any information on boundary layer flashback velocities, they do provide fundamental knowledge that is important for the general characterization of the flashback mechanism. Most importantly, all of these studies concur that radical recombination at the wall, characterized by low-activation-energy reactions, plays an important role in the FWI process and that a single-step chemistry modelling approach, lacking detailed information about radical recombination reactions, fails to predict FWI processes correctly.

Multidimensional direct simulations of FWI configurations are very expensive computationally and therefore only a few of these investigations have been reported in the literature. Moreover, because of their high cost (and in spite of the conclusions reached in the one-dimensional studies mentioned above), most of the reported multidimensional direct simulations of FWI configurations have either been restricted to a thermal-diffusive limit in which the effect of thermal expansion on the fluid 
flow is assumed to be negligible, or to a simple one-step chemical kinetics model and small physical domains. A pioneering paper by Lee \& T'ien (1982) reports a two-dimensional numerical simulation of laminar flame flashback in a sidewall quenching (SWQ) configuration and suggests that a pressure-based interaction between the premixed flame and the boundary layer flow is the reason behind a larger computed laminar flame speed in the two-dimensional configuration compared with the one-dimensional case. Subsequent studies by Kurdyumov, Fernandez \& Linan (2000) and Kurdyumov et al. (2007) and Kurdyumov \& Fernandez-Tarrazo (2002) on the boundary layer flashback of laminar two-dimensional flames added more realistic features to the model, such as effects of the fuel species Lewis number, but are still limited to one-step chemical kinetics and to the interpretation that boundary layer flashback is governed by physical processes whose main characteristics are two dimensional. Poinsot, Haworth \& Bruneaux (1993) performed a DNS of HOQ in a two-dimensional, pseudo-turbulent reactive boundary layer while Bruneaux et al. (1996) studied three-dimensional HOQ of a back-to-back, premixed flame propagating in constant-density turbulent channel flow. Again, this was performed in the thermaldiffusive limit in which there is no coupling between the flame and the flow field. Alshaalan \& Rutland (1998) and Alshaalan \& Rutland (2002) investigated the SWQ configuration for a three-dimensional, v-shaped, premixed flame anchored in weakly turbulent Couette flow and, while these numerical experiments have provided very useful statistical information, they lack the detailed chemical kinetics modelling to account for the important effects of radical recombination reactions in the immediate vicinity of the wall.

A recent study by the present authors investigated the interaction of an anchored, v-shaped, premixed $\mathrm{H}_{2}$-air flame with turbulent Poiseuille flow while employing a detailed chemical kinetics model (Gruber et al. 2010). The anchored v-flame configuration investigated in the study provides useful information about the influence of boundary layer turbulence on the flame shape and structure and on the timedependent pattern of the wall heat flux while still ensuring spatial statistical stationarity for convenient extraction of averages. However, this configuration is not well suited for investigation of the physical mechanisms behind flashback in turbulent wall boundary layers as flashback is usually characterized by a spatially unsteady, freely propagating flame front. The configuration chosen for the present DNS study consists of non-anchored premixed $\mathrm{H}_{2}$-air flames propagating upstream in the boundary layers of turbulent Poiseuille flows at two different pressures. These configurations lack spatial statistical stationarity but they provide detailed insight into the fundamental characteristics describing the dynamics of premixed flame flashback in turbulent boundary layers, thereby complementing the earlier work.

Finally, it is also important to mention that some recent experimental studies (Heeger et al. 2010; Eichler et al. 2011; Eichler \& Sattelmayer 2011, 2012; Mayer et al. 2011) have attempted to chart flashback behaviour under lean premix conditions for several different burner configurations and to shed light on the physical mechanisms behind near-wall upstream propagation of premixed flames using detailed laser diagnostics techniques with high spatial and temporal resolution. Among the important results obtained in these experiments is the conclusion that the critical velocity gradients at the wall required to avoid wall boundary layer flashback increase steeply as the pressure increases (Mayer et al. 2011).

The remainder of this paper is organized as follows: the DNS code and the problem formulation are described in $\S 2$. DNS results along with theoretical results for 


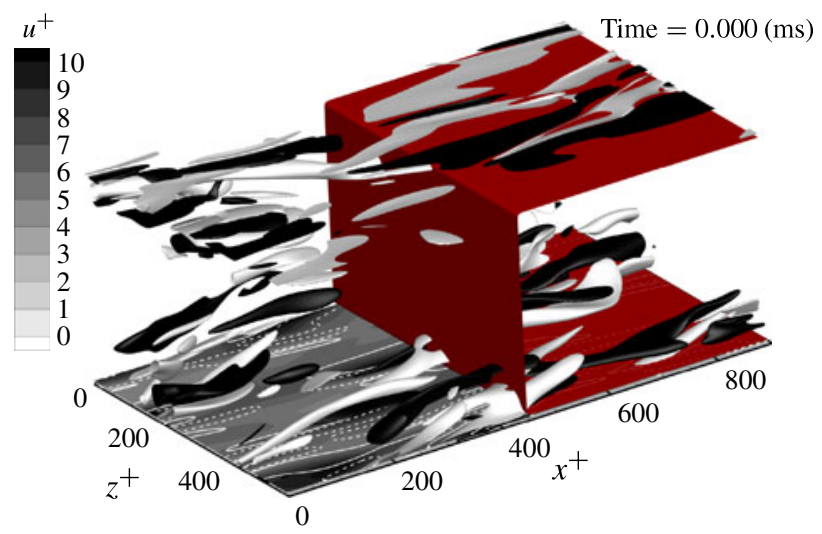

FIGURE 1. Initial conditions for the reactive case: a flat laminar flame profile (visualized by the red surface of progress variable $C=0.7$ ) is superimposed on the turbulent velocity field from the auxiliary non-reactive DNS (a number of streamwise vortices are visualized by the black and white surfaces of $x$-vorticity). The non-dimensional streamwise velocity, normalized by the friction velocity, is shown (greyscale flooded contours) on the $y^{+}=5$ plane together with the trace of $Y$-vorticity (white lines, solid and dashed lines represent opposite sign of vorticity).

estimating the occurrence of Darrieus-Landau (DL) instability are presented in $\S 3$. Finally, conclusions and recommendations for further work are presented in $\S 4$.

\section{Mathematical formulation, case configuration and DNS code}

The Navier-Stokes equations in their compressible formulation are solved in a three-dimensional computational domain to simulate the upstream propagation of a non-anchored premixed $\mathrm{H}_{2}$-air flame in fully developed turbulent channel flow at 1 and $2 \mathrm{~atm}$ (denoted as TCFP01 and TCFP02), where all other physical parameters of the reacting flows are the same.

Figure 1 illustrates the DNS configuration where an auxiliary inert turbulent Poiseuille flow is temporally sampled at a fixed streamwise position and the sampled velocity components are fed at the inlet plane of the reacting DNS. The reactive DNS is initialized by superimposing a plane laminar premixed $\mathrm{H}_{2}$-air flame in the middle of the domain onto the turbulent velocity field from the auxiliary inert DNS. The three spatial directions in this configuration are the streamwise direction $(x)$, wall-normal direction $(y)$ and spanwise direction $(z)$. The turbulent $\mathrm{H}_{2}$-air reactant mixtures enter the channel from a non-reflecting inflow boundary and approach the flame in the streamwise direction while the burnt products leave the computational domain from a non-reflecting outflow boundary. In this section the establishment of the auxiliary inert turbulent channel flow feed data and the reactive turbulent FWI DNS are described.

The chemical reactions in the gas phase are described by a detailed mechanism for hydrogen combustion in air ( $\mathrm{Li}$ et al. 2004). This mechanism consists of 9 species and 19 elementary reaction steps. Nitrogen is assumed to be inert such that $\mathrm{NO}_{x}$-formation reactions are not considered. Thermodynamic properties are modelled as polynomial functions of temperature and transport coefficients as described in the CHEMKIN and TRANSPORT packages, respectively (Kee et al. 1999). No attempt is made to incorporate the effects of radiative heat transfer in this study. 
$\operatorname{Re}_{\tau}=180$

$T_{w}=T_{u}=750 \mathrm{~K}$

$S_{l 1-2} / U_{c}^{f}=0.52-0.55$

$\delta_{l 1-2} / h=0.05$

$$
\begin{gathered}
L_{x} \times L_{y} \times L_{z}=5 h \times 2 h \times 3 h \\
\phi=1.5 \\
u_{\tau} / S_{l 1-2}=0.103-0.097 \\
x_{\text {inifl }}^{+}=450
\end{gathered}
$$

$$
\begin{gathered}
N_{x} \times N_{y} \times N_{z}=1000 \times 400 \times 600 \\
D a_{1-2}=1.19-1.22 \\
u_{\tau} / U_{c}^{f}=0.053 \\
y_{\text {iniff }}^{+}, z_{\text {inifl }}^{+}=\text {whole range }
\end{gathered}
$$

TABLE 1. DNS parameters for the reactive cases: $h$ is the channel half-width and the suffix inifl refers to the flame surface initialization.

The premixed $\mathrm{H}_{2}$-air mixtures in the approaching flows are characterized by an equivalence ratio, $\phi$, equal to 1.5 while the temperature of the wall and of the reactants is set to $750 \mathrm{~K}$ for both TCFP01 and TCFP02. The pressure in the entire domain is initialized to $1 \mathrm{~atm}$ in TCFP01 and to $2 \mathrm{~atm}$ in TCFP02. Although the fuel-rich mixture and the relatively low pressures employed do not represent the nominal operating conditions of lean premixed combustion in stationary gas turbines, these choices result in relatively high flame speed, allowing high approach flow velocity, short channel transit time and, ultimately, lower computational cost for the DNS. The DNS provides extremely detailed fundamental knowledge about the physical mechanism controlling boundary layer flashback of turbulent premixed flames.

The Reynolds number of the approach flow is $R e_{0} \sim 3200$ for both TCFP01 and TCFP02, based on the channel centreline velocity $U_{c}^{f}$ of the fresh reactants and the channel half-widths, $h_{1}$ and $h_{2}$ respectively $\left(h_{2}=h_{1} / 2=0.5 \mathrm{~cm}\right)$, where subscripts 1 and 2 denote cases TCFP01 and TCFP02, respectively. This corresponds to a friction Reynolds number $\operatorname{Re}_{\tau} \sim h / \delta_{v} \sim 180$, where $\delta_{v}$ is the viscous length scale. All turbulent quantities used below for non-dimensionalization characterize the turbulent flow of the fresh reactants upstream of the flame. The Damköhler numbers (ratio of a chemical to dynamical time scale) that characterize the turbulent flames are $D a_{1} \sim 1.19$ and $D a_{2} \sim 1.22$. As suggested by Gruber et al. (2010), these Damköhler numbers are based on the freely propagating one-dimensional laminar flame time scale $\left(t_{l 1}=\delta_{l 1} / S_{l 1} \sim 3.7 \mathrm{e}^{-05} \mathrm{~s}\right.$ and $\left.t_{l 2}=\delta_{l 2} / S_{l 2} \sim 1.8 \mathrm{e}^{-05} \mathrm{~s}\right)$ and on the wall time scale that is uniquely defined from the turbulent channel flow of the fresh reactants $\left(t_{w 1}=v_{1} / u_{\tau}^{2} \sim 4.4 \mathrm{e}^{-05} \mathrm{~s}\right.$ and $\left.t_{w 2}=v_{2} / u_{\tau}^{2} \sim 2.2 \mathrm{e}^{-05} \mathrm{~s}\right)$. In these expressions $u_{\tau}$ is the friction velocity, $v_{1}$ and $v_{2}$ are the kinematic viscosities of the fresh reactants, and $\delta_{l 1,2}$ and $S_{l 1,2}$ are the laminar flame thicknesses and laminar flame velocities at the two pressure levels. It should be noted that the flame thicknesses $\delta_{l 1,2}$ at 1 and 2 atm, respectively, are expressed in term of the thermal flame thickness defined as $\delta_{l}=\left(T_{b}-T_{u}\right) / \max (|\partial T / \partial x|)$, where $T_{u}$ and $T_{b}$ are the temperatures of unburned and burned gases, respectively, and $\max (|\partial T / \partial x|)$ is the maximum temperature gradient (Poinsot \& Veynante 2001). Other relevant parameters of the DNS are given in tables 1 and 2. Note that the non-dimensional centreline velocity is $u_{c}^{+} \sim U_{c}^{f} / u_{\tau} \sim 19$.

An auxiliary DNS of fully developed, non-reacting turbulent plane channel flow (case TCFAUX) driven by an effective streamwise pressure gradient is used to provide the reactive cases with an initial turbulence field and inlet turbulence. The velocity fluctuations imposed at the channel inlet of the reactive case are obtained by temporal sampling of the temporally evolving turbulence at a fixed streamwise location in the auxiliary inert simulation. This approach, which permits eddies to 'evolve' on the boundary, provides a more realistic description of the incoming turbulence compared with the usual practice of convecting a turbulence field at an instant into the domain by Taylor's hypothesis. See Gruber et al. (2010) and Grout et al. (2012) for details 


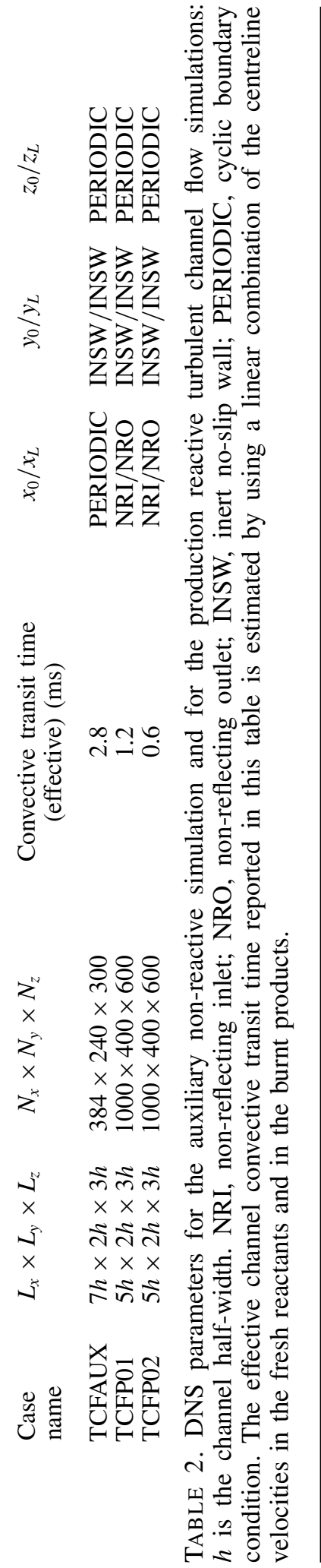




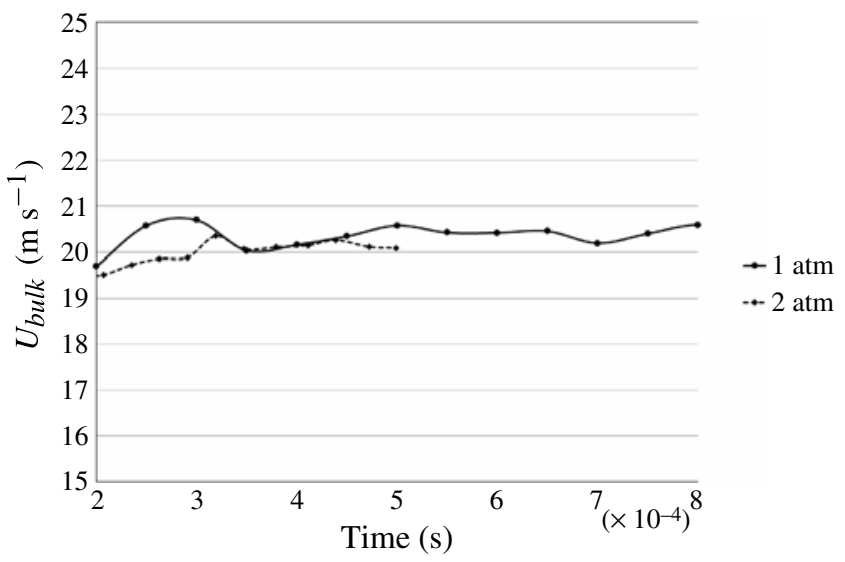

FIGURE 2. Time evolution of the actual channel bulk velocity sampled at the inlet plane for cases TCFP01 and TCFP02.

about the validation of the present DNS code in both zero and non-zero pressure gradient turbulent boundary layer flows.

Navier-Stokes characteristic boundary conditions (NSCBCs) are implemented based on the original formulation of Poinsot \& Lele (1992) and on the later improvements described in Sutherland \& Kennedy (2003), Yoo et al. (2005) and Yoo \& Im (2007). The boundary conditions are non-reflecting at the inflow $(x=0)$ and outflow $\left(x=L_{x}\right)$ planes (thereby ensuring that spurious pressure waves can exit the domain), no-slip isothermal solid surface at the wall boundaries $\left(y=0\right.$ and $\left.y=L_{y}\right)$, and periodic in the spanwise direction $\left(z=0\right.$ and $\left.z=L_{z}\right)$. The present NSCBC implementation allows only small variations around the nominal values of the time-varying velocity components imposed at the inlet plane, figure 2 illustrates the time evolution of the instantaneous values of the actual bulk velocities imposed at the channel inlet for cases TCFP01 and TCFP02. The wall is assumed to be impermeable, so the wall-normal mass flux of all chemical species is set to zero.

The reactive cases TCFP01 and TCFP02 are initialized by imposing a constant pressure equal to 1 and $2 \mathrm{~atm}$, respectively, throughout the domain and by imposing the instantaneous fluctuating velocity, density and temperature fields computed in the inert simulation (see figure 1). This procedure ensures that the flame encounters realistic approaching turbulence from the beginning of the simulation, thereby enabling a relatively short settling time. A planar premixed laminar flame placed in the middle of the domain is superimposed on the initial velocity field obtained from the auxiliary non-reacting simulation. Burnt adiabatic product conditions are imposed downstream of the flame except very close to the wall, see below. The initial streamwise velocity field component in the reacting simulations is adjusted, only once and prior to the start of the simulation, for compatibility with the lower density on the product side of the flame. A progress variable function $C$ is used in the initialization to map all points in the three-dimensional domain to a one-dimensional CHEMKIN PREMIX (Kee et al. 1999) solution of the freely propagating $\mathrm{H}_{2}$-air flame configuration. The progress variable $C$ is a scalar parametrization of the reactive flow field, based on the hydrogen mass fraction, that is equal to zero in the fresh reactants and unity in the burnt products. Note that the initialization method used here (based on $C$ ) results in a U-shaped, marginally incorrect, flame surface, i.e. the two flame branches extending 
along the walls (see figure 1) are a direct consequence of the $C$-parametrization and of the fact that the wall boundaries are isothermal and kept at the fresh reactants' temperature. Moreover, this initialization technique yields a marginally incorrect initial pressure field. Therefore, an initial 'settling' time interval of approximately five times the channel acoustic transit time $\left(7 h_{1-2} / c=7.36-3.68 \mathrm{e}^{-05} \mathrm{~s}\right)$ is required for the initial pressure fluctuations to exit the domain from the inlet and outlet boundaries. After an initial settling time interval $\Delta t^{s}$ approximately equal to $3.7 \mathrm{e}^{-04} \mathrm{~s}$ for TCFP01 and $1.9 \mathrm{e}^{-04} \mathrm{~s}$ for TCFP02, the turbulence-flame interaction is no longer affected by the initial pressure fluctuations and at this point the flames have been wrinkled by the approaching turbulence and have begun to propagate upstream.

Owing to the intrinsic transient characteristics of this particular flame configuration, results are sampled every 0.5 wall time units, $t_{w}$, to ensure sufficient sample sizes for statistical post-processing. The sampling interval is equivalent to approximately one channel convective transit time, as reported in table 2, providing a total of 44 samples after the initial transient and before the flame reaches the region near the upstream inlet boundary of the domain. The numerical integration time step is fixed at a value of $\Delta t=4.0 \mathrm{e}^{-09} \mathrm{~s}$ in the reactive case and at a value of $\Delta t=1.0 \mathrm{e}^{-08} \mathrm{~s}$ in the inert auxiliary simulation, corresponding to 9000 and 4500 time steps per wall time unit, $t_{w}$, respectively.

The three-dimensional Cartesian grid is uniform in all directions. The first point from the wall is at $y^{+}=0.8$ where the superscript + indicates non-dimensionalization by the viscous length scale. There are 11 points within $y^{+}=10$ to satisfy the resolution requirements in the viscous layer (Moser, Kim \& Mansour 1999). The grid resolution is $\Delta x^{+}=\Delta y^{+}=\Delta z^{+}=0.8$ (equivalent to $50 \mu \mathrm{m}$ for TCFP01 and $25 \mu \mathrm{m}$ for TCFP02). The grid is not stretched, not even in the wall-normal direction, in order to accurately represent the flame which requires high spatial resolution throughout the channel, including near the centreline.

The parallel DNS code, S3D (Chen et al. 2009), is used to perform the present DNS. In addition to the previous FWI study (Gruber et al. 2010), S3D has been used for a range of studies, including premixed flames (Hawkes \& Chen 2005; Sankaran et al. 2007), non-premixed flames (Hawkes et al. 2007; Yoo, Sankaran \& Chen 2009; Grout et al. 2011, 2012; Kolla et al. 2012) and auto-ignition (Echekki \& Chen 2003; Sankaran et al. 2005).

S3D is written in FORTRAN 90 and uses the Message Passing Interface (MPI) for interprocess communication in parallel execution. The algorithm implemented in S3D solves the Navier-Stokes equations for a compressible fluid in conservative form on a structured, Cartesian mesh in one, two or three spatial directions. Spatial derivatives are computed with an eighth-order, explicit, centred, finite-difference scheme (thirdorder one-sided stencils are used at the domain boundaries in the non-homogeneous directions) in conjunction with a tenth-order, explicit, spatial filter, as described in Kennedy \& Carpenter (1994), to remove high-frequency noise and reduce aliasing error. A fourth-order, six-stage, explicit Runge-Kutta scheme, described in Kennedy, Carpenter \& Lewis (2000), is used for time integration.

The non-reactive auxiliary DNS and the reactive production DNS presented here were run on 512 and 12000 processor cores, respectively (for a total computational cost of 1.4 M CPUhrs), the former on SINTEF Energy Research's own HPC cluster and the latter on the JAGUARPF architecture that is part the National Center for Computational Science at Oak Ridge National Laboratory (ORNL). JAGUARPF is the CrayXT5 2.3 petaFLOPS supercomputer at the ORNL Leadership Computing Facility (OLCF). 


\section{Results}

In this section DNS results from simulations of turbulent FWI configurations with flashback are presented. First, instantaneous plots of relevant flow and reaction quantities are presented to identify the unsteady upstream propagation mechanism for premixed flames in turbulent boundary layers; then, estimates for the upstream flashback flame propagation velocity in turbulent boundary layers are presented and compared for both cases TCFP01 and TCFP02. Please note that the flames propagate upstream against nominally equal bulk flow velocities for both pressure levels as shown in figure 2. Finally, the effect of flame shape and distance from the wall on the flame heat release rate is investigated.

\subsection{Upstream flame propagation}

The reactive DNSs are initialized at $t=0.0 \mathrm{~s}$ by superimposing fresh reactants' and burnt products' fields (separated by a flat laminar flame region) onto the turbulent velocity field from a non-reactive auxiliary DNS. The transient that follows provides consistent flow, temperature, density and composition fields within the first $\Delta t_{1-2}^{s} \sim 0.4-0.2 \mathrm{~ms}$. After this initial 'settling' time, sampling of the data for analysis commences and the unsteady evolution of the flame is studied until the flame reaches the immediate vicinity of the upstream boundary of the domain. There, the flame-flow interaction is distorted by the imposed time-dependent inflow boundary conditions. Figure 3 illustrates several stages of the unsteady flame propagation along the channel walls for TCFP02 (the qualitative FWI behaviour observed in TCFP01 is identical). Note that the upper wall is not shown and that the surfaces visualized represent:

(i) the streamwise velocity normalized by the friction velocity $u^{+}=u / u_{\tau}$ on the $y^{+}=5$ plane (greyscale flooded contours on the plane parallel to the wall);

(ii) the wall-normal vorticity $\omega_{y}$ on the $y^{+}=5$ plane denoting the streamwise vorticity structures of the boundary layer (white lines on the plane parallel to the wall, solid and dashed lines denote opposite sign);

(iii) the flame surface as described by the progress variable isosurface, $C=0.7$ (red isosurface);

(iv) back-flow regions characterized by negative streamwise velocity and located upstream of the flame surface portions that are convex towards the reactants (blue isosurfaces).

Note that the back-flow regions of negative streamwise velocity are completely absent in the initial flow field taken from the non-reactive simulation, and hence one can assume that their presence is a direct consequence of the interaction of the flow field with the premixed flame. Moreover, it is interesting to observe, in the fresh reactants' stream, how the high-speed sweeps, located in between the streamwise vortex pairs and highlighted by the dark grey areas on the plane parallel to the wall, cause an initial local displacement of the flame surface downstream of its original position. These sweeps ultimately induce the formation of several flame cusps towards the products' side. Conversely, the initial deformation of the flame sheet into bulges that are convex towards the reactants is initiated by the presence of low-speed ejections approaching the flame region. Locally this allows some portions of the flame to propagate upstream. Furthermore, once the formation of these bulges and cusps is initiated by the approaching fluctuating velocity field, they induce a DL hydrodynamic instability (Williams 1985) that, in turn through its feedback effect on the velocity and pressure fields, further enhances the flame wrinkling in a continued interaction with 
(a)

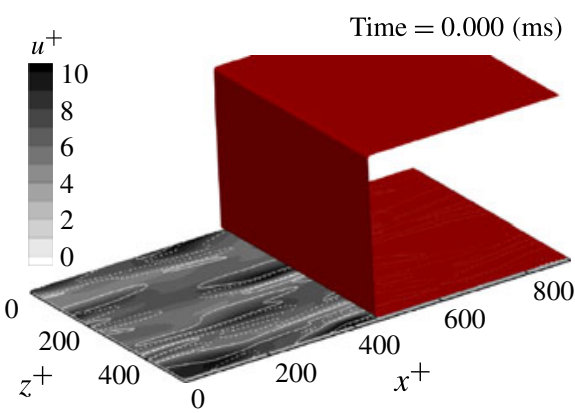

(c)

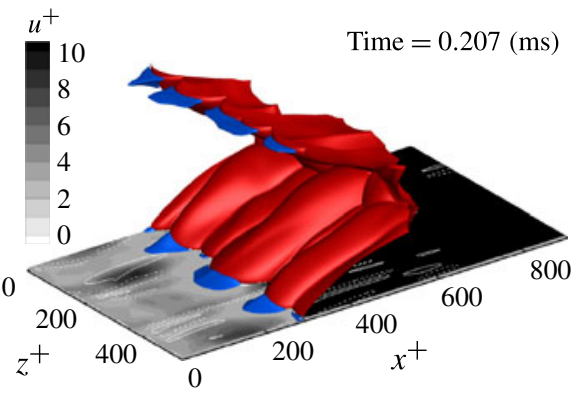

(e)

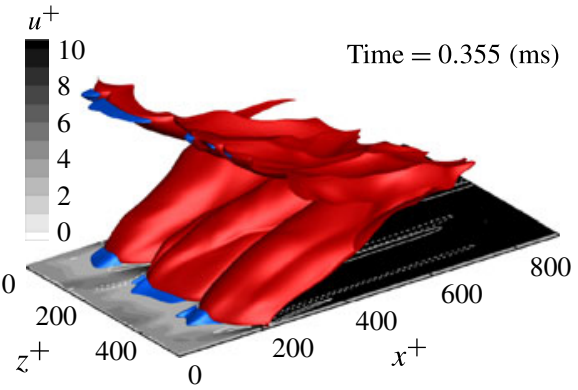

$(g)$

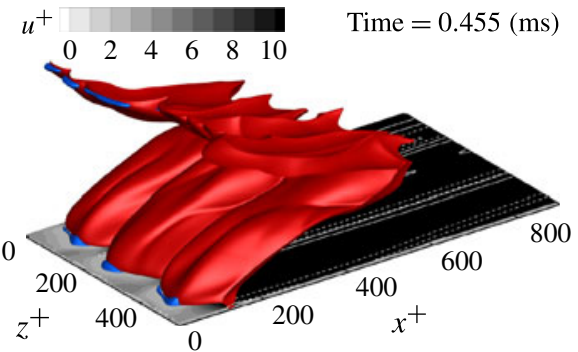

(b)

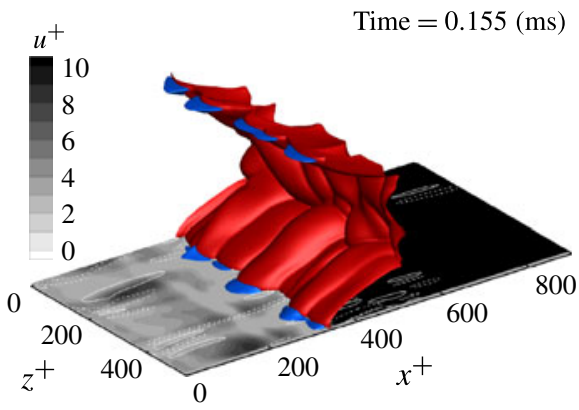

(d)

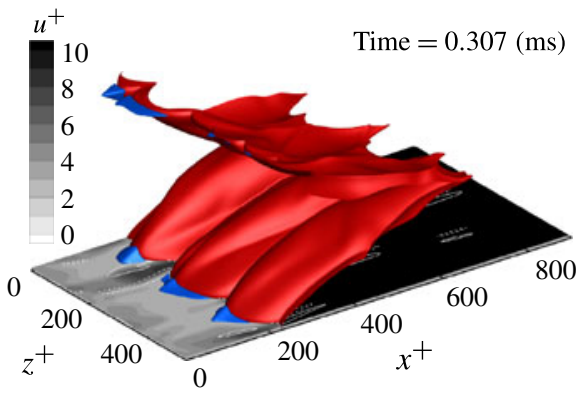

$(f)$

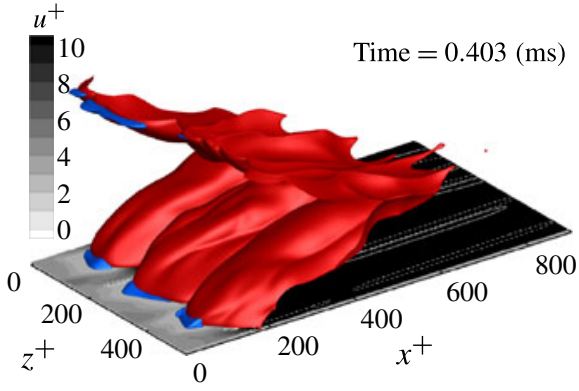

( h)

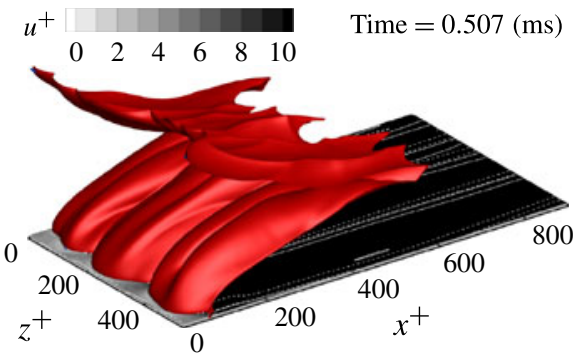

FIgURE 3. Temporal evolution of the premixed flame $(C=0.7)$, red isosurface, and of the back-flow regions, blue isosurfaces, from the beginning to the end of the reactive simulation TCFP02. The non-dimensional streamwise velocity (greyscale flooded contours) is shown on the $y^{+}=5$ plane together with the trace of $Y$ vorticity (white lines, solid and dashed patterns represent opposite sign).

the approaching turbulent flow field (see §3.4). In the following discussion the term flame bulge refers to a portion of the wrinkled flame surface that is convex towards the reactants and characterized by a relatively large radius of curvature, while the term 

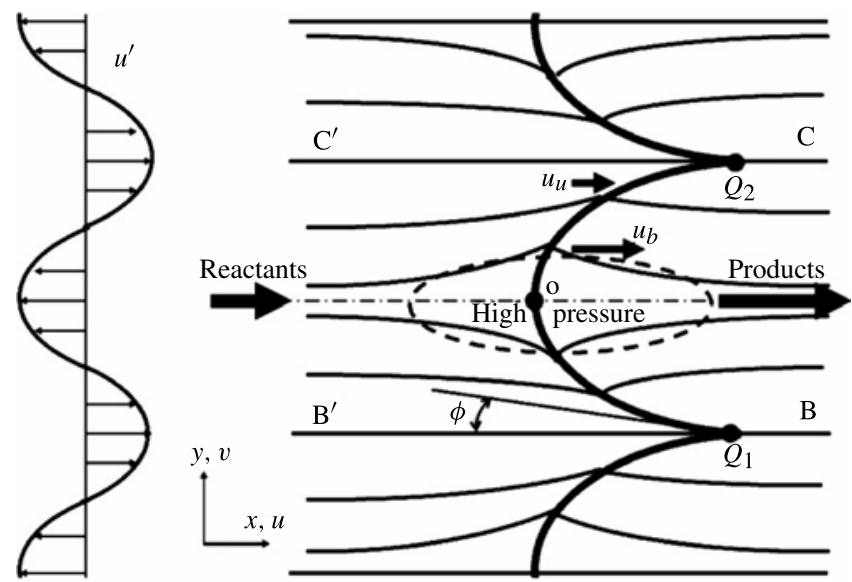

FIGURE 4. Schematic of the DL hydrodynamic instability as presented by Lipatnikov \& Chomiak (2010): an elementary turbulent flame generated by a single shear wave that represents turbulence. Straight streamlines $B^{\prime} B$ and $C^{\prime} C$ cut out a representative flame segment. (Reproduced with permission from Lipatnikov \& Chomiak (2010).)

flame cusp refers to a portion of the flame surface that is convex towards the products and characterized by a relatively small radius of curvature.

Observations from the DNSs suggest that the interaction between the turbulent boundary layer flow field and the premixed flame geometry is responsible for the generation of the highly localized back-flow regions of the flow (blue surfaces) that are clearly visible immediately upstream of each flame bulge (and only there) and that are limited to the near-wall region $\left(y^{+}<20\right)$. The physical mechanism behind the formation of these back-flow regions is likely to have its origin in the DL instability wherein the geometrical characteristics of the wrinkled flame surface induce changes in the streamline pattern and affects the near-wall pressure field. This, in turn, induces a positive pressure gradient immediately upstream of the flame bulges and ultimately causes a flow reversal. In a recent comprehensive review by Lipatnikov \& Chomiak (2010) the effect of premixed flames on turbulent flows is discussed and in particular the intrinsic hydrodynamic DL instability and its effect on the flow field is described. Figure 4 is reprinted from that paper and provides a schematic representation of the DL instability for the general case of unbounded flow. The principal difference between the present case and the DL instability acting on unbounded turbulent flows is that the fluid approaching a flame bulge would only be retarded in the latter unbounded flow case while, for the slow fluid flowing very close to the wall, a flow reversal is induced.

An additional interesting observation is that, when the flame surface propagates very close to the upstream inlet, where time-dependent, positive streamwise velocities are specified as a boundary condition, the back-flow regions become smaller and weaker figure $3(\mathrm{~g})$, eventually disappearing completely figure $3(h)$ as the flame reaches the domain boundary. The disappearance of the back-flow regions, caused by the finite size of the computational domain, induces a decrease in the flame propagation velocity, see $\S 3.5$ for a more detailed discussion of this observation.

Finally, it is important to note that the very same features of near-wall premixed flame propagation shown here, including the flame-induced negative streamwise 
velocity (back-flow) regions, have also been observed experimentally utilizing high resolution diagnostics (Eichler \& Sattelmayer 2012). The laboratory experiments and the present numerical simulations are conducted in a coordinated effort to study boundary layer flashback within the framework of BIGCO2/BIGCCS. However, the two studies are not directly comparable, apart from the qualitative observation that back-flow regions are observed in both, as the present simulations are representative of rich stoichiometric conditions while the laboratory experiments were conducted for very lean fuel-oxidant mixtures. Eventual local enrichment effects due to differential diffusion of the deficient reactants (particularly important effect for globally lean flames) are likely to contribute to local variations in the flame speed along the curved flame surface. This would further increase the flame wrinkling and global propagation velocity, but does not appear to impact the formation of the back-flow regions that are observed for both lean and rich conditions. A future DNS study will consider the same fuel-lean $\mathrm{H}_{2}$-air mixture as the experiment, and by increasing the computational domain size, will also facilitate the passage of multiple boundary layer streaky structures interacting with the flame.

Summarizing, the transient upstream flame propagation (flashback) described herein is characterized by the following sequence of events.

(i) Initially flat laminar shape and absence of regions characterized by negative streamwise velocity at $t=0.0 \mathrm{~s}$, see figure $3(a)$.

(ii) Initial wrinkling of the flame while it is still affected by the marginally unphysical pressure and velocity fields during the initial transient, see figure $3(b)$. Note the visible signature on the velocity field of a transverse pressure wave normal to the bulk flow direction.

(iii) Freely propagating flame moving upstream along the channel wall and the appearance of back-flow regions immediately upstream of the flame bulges, see figure $3(c-f)$.

(iv) Approach of the flame in the vicinity of the upstream domain inlet and effect of the boundary conditions' specification on the back-flow regions' size, see figure $3(g)$, ultimately leading to their disappearance, see figure $3(h)$.

\subsection{Back-flow regions}

We now study the features of the flame-induced, near-wall back-flow regions whose presence immediately upstream of localized regions of a propagating premixed flame has not been reported in previous modelling studies. The experimental studies by Heeger et al. (2010) and Eichler \& Sattelmayer (2012) were the first to report this phenomenon. Figure 5(a) focuses on selected representative flame segments showing the spatial variation in the pressure field and illustrating the presence of a relatively high-pressure region immediately upstream of the flame bulges and, by comparison, a relatively low-pressure region upstream of the flame cusp. Figure 5(b) further shows that the high-pressure region at the flame bulge extends from the wall to $y^{+} \sim 15$. Figure 6(a) shows the streamwise velocity field in an $x z$-plane at $y^{+}=10$ above the wall while figure $6(b)$ shows the corresponding spanwise velocity field and the streamlines on the $y^{+}=10$ plane. The figures highlight the effect of the flame geometry on the approaching reactant flow. In particular, the flame geometry induces a characteristic divergent-convergent streamline pattern and a quadrupole pattern in the spanwise velocity field, denoted by the letters A, B, C and D in figure $6(b)$. Furthermore, figure $6(a)$ also shows the role of the high-velocity 
(a)

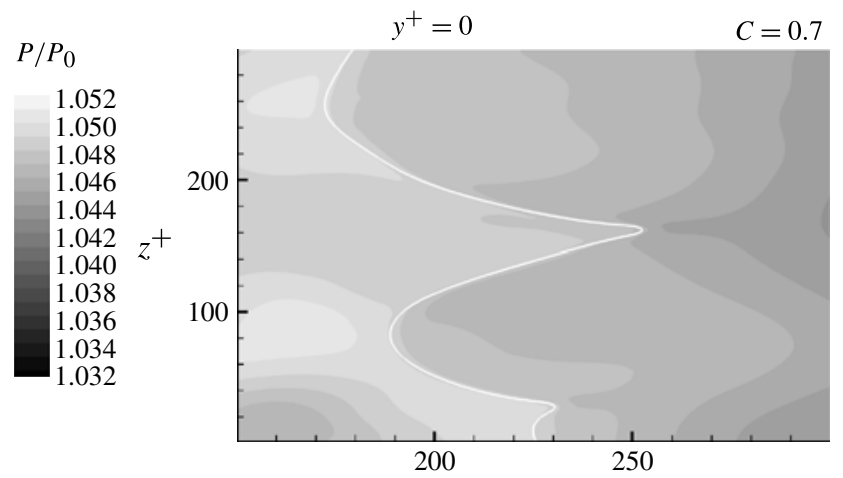

(b)

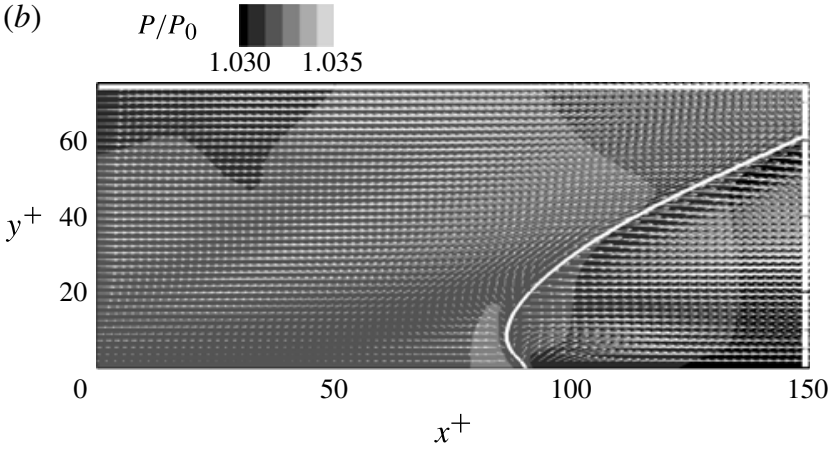

FIGURE 5. Instantaneous pressure field (normalized by the initial pressure $P_{0}$ ) at the wall at $y^{+}=0$ shown in a $x z$-plane highlighting a representative flame segment $(a)$, the high-pressure region immediately upstream of the flame bulge and the low-pressure region immediately upstream of the flame cusp. Details of the pressure field are also shown on a $x y$-plane $(b)$ oriented normal to both the wall and the flame bulge at its most upstream position (point ' $\mathrm{O}$ ' in the idealization of figure 4). The flame is denoted by the reaction progress variable $C=0.7$ in both figures (white solid line), where unburnt mixture is on the left and burnt mixture is on the right of the lines.

sweeps of the turbulent boundary layer in causing flame wrinkling and inducing the formation of flame cusps by regions of high streamwise velocity, elongated in the streamwise direction, that end up in the narrow cusps of the flame surface. The bluecoloured area of negative streamwise velocity visible in figure $7(a, b)$ quantifies the maximum strength of the flame-induced back-flow region to $u^{+} \sim-5$ or, equivalently, approximately half of the laminar flame speed $S_{l 2}$. This occurs at a wall distance $\left(y^{+} \sim 9\right)$ that is well above the quenching distance for this particular flame (see $\S 3.6$ ) and where, in the absence of the flame, the boundary layer streamwise velocity would have been of the order of $u^{+} \sim 6$. Thereby, a considerable streamwise velocity difference $\Delta u^{+} \sim 11$ is encountered by the reactant flow immediately upstream of the flame, resulting in a relatively high flame propagation velocity along the wall (see $\S 3.5)$. Note that the plots of figure $7(a, b)$ are qualitatively similar to the plots presented in Eichler \& Sattelmayer (2012) and obtained from the laminar and turbulent flashback experiments and to the plots from the simplified modelling approach (twodimensional laminar boundary layer, reduced finite rate chemistry) presented therein to complement the experiments. The overall size and shape of the backflow regions observed experimentally are correctly reproduced by the DNSs. Roughly triangular in 


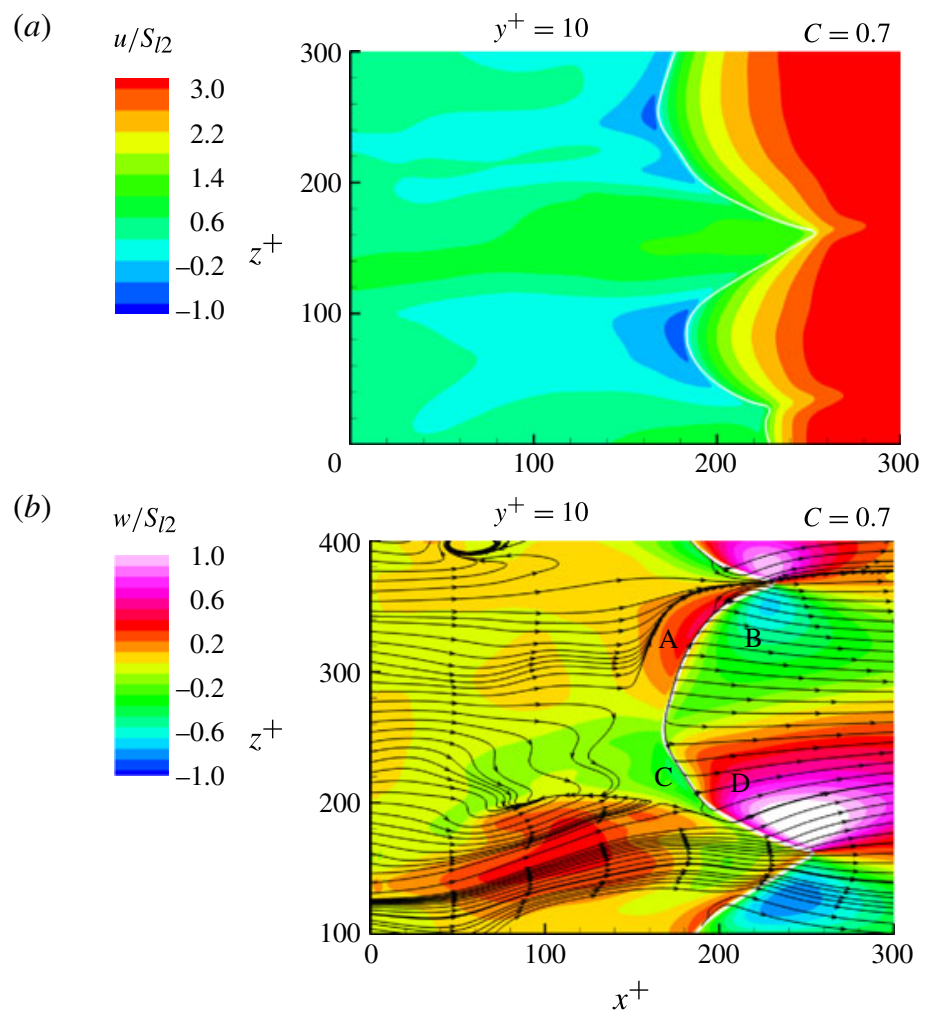

FIGURE 6. Spatial variation of the in-plane streamwise $(a)$ and spanwise $(b)$ velocity fields normalized by the laminar flame speed $S_{l 2}$ shown in a $x z$-plane above the wall at $y^{+}=10$. The flame is denoted by the reaction progress variable $C=0.7$ in both figures (white solid line), where unburnt mixture is on the left and burnt mixture is on the right of the lines. The quadrupole flow pattern at the flame bulge is marked by the letters $\mathrm{A}, \mathrm{B}, \mathrm{C}, \mathrm{D}$ in $(b)$ and the streamline pattern is denoted by black arrowed lines.

shape, these backflow regions extend up to $y^{+} \sim 20$ away from the wall (closest to the flame) and about 60-70 wall units in the upstream direction.

Figure 8 shows the one-dimensional streamwise profiles of some relevant quantities immediately above the wall, at $y^{+}=2$, corresponding to a representative flame bulge (figure $8 a$ ) and flame cusp (figure $8 b$ ). The most important difference between the two figures is the existence of an adverse pressure gradient associated with a negative velocity gradient at the flame bulge. In contrast, a positive pressure gradient associated with a positive velocity gradient exists at the flame cusp.

Concluding the present section, it is important to note that intentionally underresolved tests (not shown) were performed utilizing the same DNS code and coarser grids (suboptimal in the DNS context). The tests, performed at coarser resolutions, equal to $1 / 2$ and $1 / 3$ of the production case resolution, indicate that the size and strength of the backflow regions are strongly affected by the near-wall resolution, with decreasing resolution leading to a weakening of the flow reversals at the wall and ultimately to their disappearance for the coarsest grid tested. Therefore, large Eddy simulation (LES) approaches that do not resolve the wall boundary layer nor adequately account for the flame feedback on the flow field in the subgrid scale 
(a)
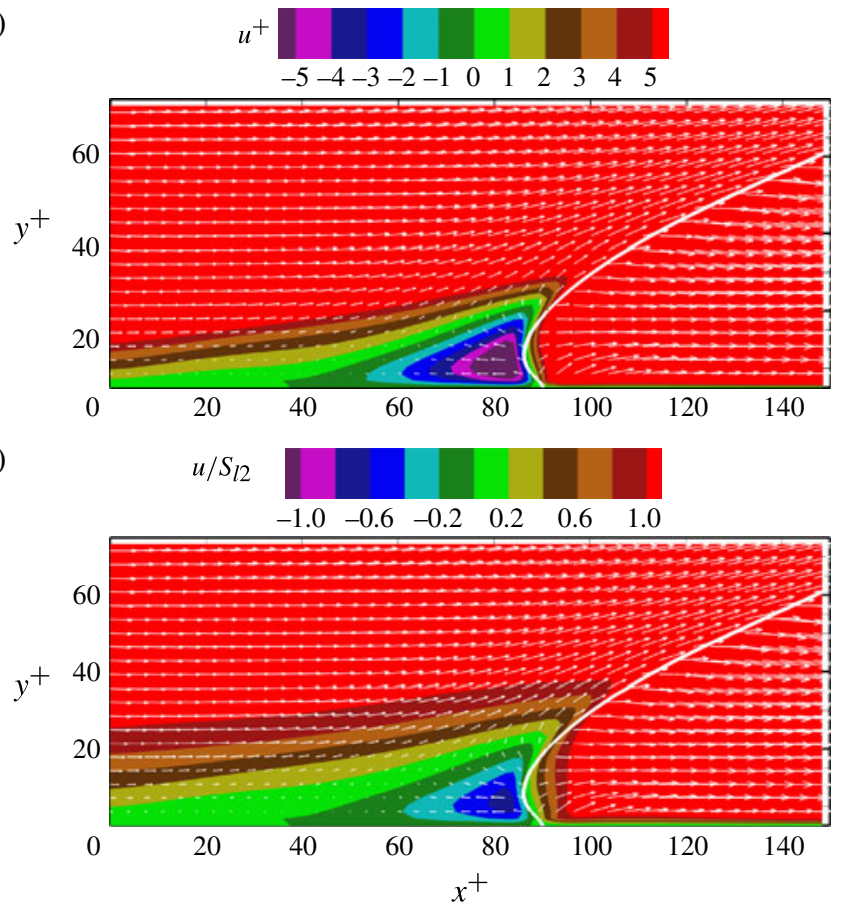

FIGURE 7. The spatial variation of the streamwise velocity field is also given on a $x y$-plane oriented normal to both the wall and the flame bulge at its most upstream position (point ' $\mathrm{O}$ ' in the idealization of figure 4). The streamwise velocity is normalized by the friction velocity $(a)$ and by the laminar flame speed $(b)$. The flame is denoted by the reaction progress variable $C=0.7$ in both figures (white solid line), where unburnt mixture is on the left and burnt mixture is on the right of the lines.

model, will likely fail to predict the presence of the backflow regions described above. This has obvious implications for their ability to accurately predict the occurrence of boundary layer flashback.

\subsection{Boundary layer flashback versus duct centerline flashback}

Finally, it is worthwhile to briefly discuss the important role of the mechanisms that lead to flashback into the approaching reactant flow field and on the formation of the backflow regions described above. Figure $9(a)$ shows the pointwise time-averaged streamwise velocity field for the anchored v-flame configuration described in Gruber et al. (2010). In that DNS study, near-wall regions of reverse flow located ahead of the flame sheet were not observed in the instantaneous velocity fields. The anchored vflame configuration places the most upstream flame portion near the channel centreline and the flame shape in relation to the duct geometry results in convergent streamlines in the fresh reactants near the wall. This, in turn, results in streamwise acceleration of the near-wall reactants flow and ultimately in thinner boundary layers. The streamwise acceleration of the reactants in the vicinity of the wall and the thinner boundary layers diminish the likelihood of flow reversal in the near-wall regions for the anchored v-flame configuration. On the other hand, figure $9(b)$ shows that the shape of the freely propagating flame results in a completely different flow field in the fresh reactants characterized by streamwise acceleration near the channel centreline, thickening of 
(a)

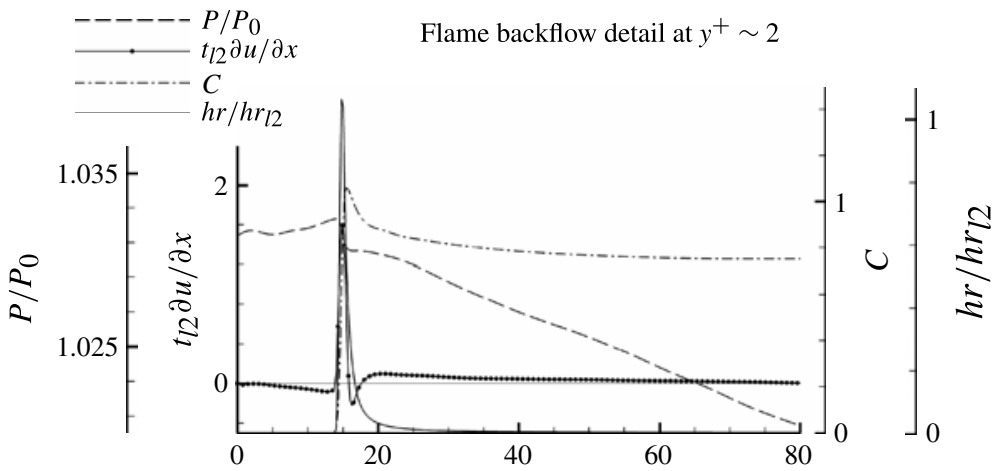

(b)

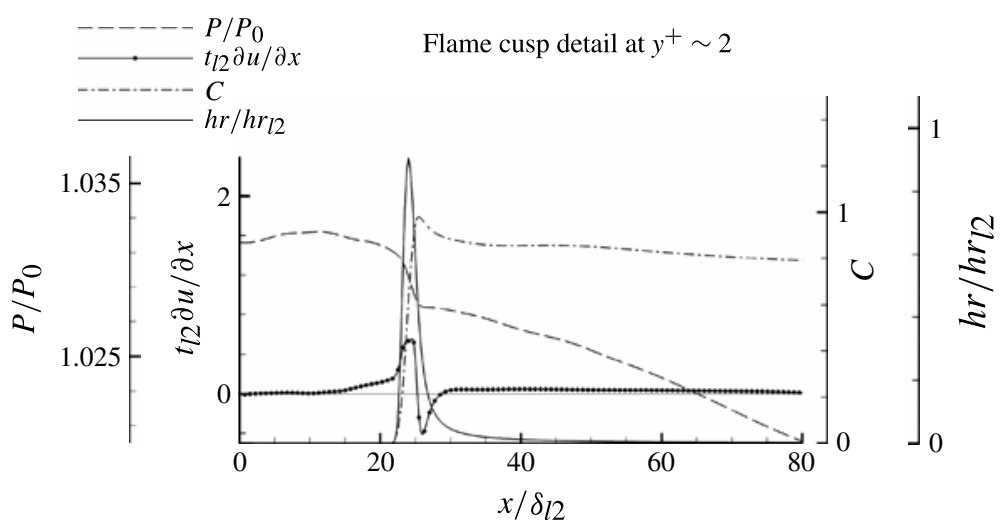

FIGURE 8. Streamwise one-dimensional profiles of pressure (normalized by its initial value), streamwise velocity gradient (normalized by the laminar flame time $t_{12}$ ), reaction progress variable and heat release rate (normalized by the laminar value $h r_{l 2}$ ). The profiles correspond to cuts across the flame at the most upstream $(a)$ and most downstream $(b)$ locations (corresponding respectively to point ' $\mathrm{O}$ ' and ' $Q_{1}$ ', or ' $Q_{2}$ ', in the idealization of figure 4). The spatial extent of the profiles is normalized by the laminar flame thickness $\delta_{l 2}$.

the boundary layers and the presence of backflow regions immediately ahead of the flame. Note, however, that the average quantities shown in figure $9(b)$ correspond to a very limited number of samples as this transient configuration does not allow the accumulation of pointwise time-averaged statistics over a long time period. Also, it is important to consider that even if both cases are characterized by the same Reynolds number for the approaching reactant flow, $R e_{\tau}$, the channel centreline velocities for the anchored and freely propagating flame cases are 80 and $25 \mathrm{~m} \mathrm{~s}^{-1}$, respectively.

The discussion above suggests that, as an important issue for applications, flashback initiated by upstream flame propagation near the duct centreline, e.g. flashback due to combustion-induced vortex breakdown in a swirl burner (Fritz et al. 2004), is unlikely to cause near-wall, flame-generated backflow regions to form. In the case of combustion-driven vortex breakdown the flame present in the core flow will lead to converging streamlines near the wall, which in turn will lead to a stabilizing effect there, preventing near-wall upstream flame propagation. Conversely, upstream flame propagation that is initiated near solid surfaces, e.g. close to the edges of a premixing duct, will likely lead to the formation of near-wall regions of reverse flow ahead of 
(a)

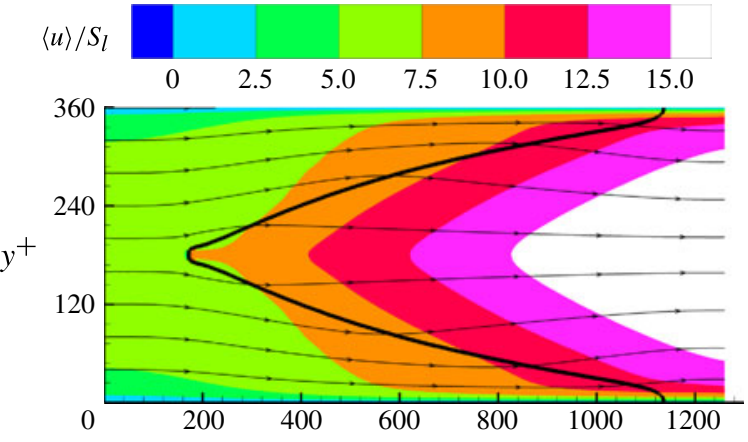

(b)

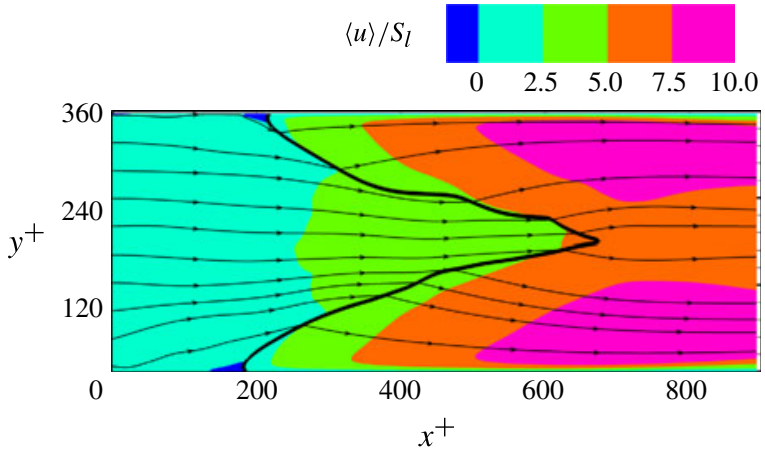

FIGURE 9. Averaged streamwise velocity field normalized by the laminar flame speed for the anchored v-flame configuration described in Gruber et al. (2010) (a) and for the present freely propagating flame configuration $(b)$. The flames are denoted by thick black lines corresponding to reaction progress variable $C=0.7$, and the streamlines by thin black lines with arrowheads in both figures.

the flame sheet, resulting in thickened boundary layers, high streamwise velocity of the reactants near the duct geometrical centre and ultimately high flashback speeds.

\subsection{Estimates of relative contribution to flame front wrinkling in the near-wall region} from DL instability and approaching turbulence

The characteristic pressure and velocity fields described above are a direct consequence of the streamline patterns initially induced on the approaching flow by flame front wrinkling and the subsequent flame acceleration. For the rich fuel-oxidant composition used in the present DNS $\left(\mathrm{H}_{2}-\right.$ air at $\left.\phi=1.5\right)$, the influence on flame wrinkling of cellular instabilities is inactive. Consequently only two mechanisms contribute directly to flame front wrinkling: turbulence-flame interaction and DL hydrodynamic instability. In order to assess the relative importance of turbulence and DL instability on the flame front wrinkling, a number of quantitative estimates are conducted below.

Lipatnikov \& Chomiak (2010) stated that flame-generated hydrodynamic effects on the flow field are not able to accelerate the flame for situations where $S_{l} \ll u^{\prime}$. However, in the present configuration, the no-slip condition at the wall ensures that $S_{l} \sim u^{\prime}$. It is therefore conceivable that, in the near-wall region, the hydrodynamic DL instability may contribute to flame acceleration, particularly in the initial phase of flame propagation. In fact, it is reasonable to assume that the streaky pattern of sweeps and ejections of the turbulent boundary layer, with alternating regions of high 
and low streamwise velocity, is responsible for initially triggering the DL instability by wrinkling the initially flat flame surface. Moreover, several studies have also suggested that turbulent fluctuations, acting over a range of time and length scales, may damp the DL hydrodynamic instability (Lipatnikov \& Chomiak 2010; Chaudhuri, Akkerman \& Law 2011; Creta, Fogla \& Matalon 2011). We have therefore performed a quantitative analysis to estimate and compare the time and length scales associated with the oncoming turbulence and with the DL instability.

For the present fuel-oxidant mixture at a pressure of $2 \mathrm{~atm}$, the resulting flame thickness is $\delta_{l 2}=0.05 h=2.5 \times 10^{-4} \mathrm{~m}$ (the subscript 2, indicating $P=2 \mathrm{~atm}$, is removed from all quantities employed in the remainder of the present section). Recall that $\delta_{l}$ is a thermal flame thickness (Poinsot \& Veynante 2001) and that reconstructing an effective characteristic, or diffusive, flame thickness $\delta_{L}$ from the thermal thickness (Blint 1986), we obtain an estimated $\delta_{L} \approx 6.0 \times 10^{-5} \mathrm{~m}$ that can be used for analysis of the DL hydrodynamic instability (Law 2006).

The DL cut-off wavelength $\lambda_{c}$ is proportional to the characteristic flame thickness $\delta_{L}$ and can be estimated using the following equation, as explained in Bychkov \& Liberman (2000):

$$
\begin{aligned}
\lambda_{c} & =\delta_{L} \frac{\pi(\Theta-1)}{\Gamma \sqrt{\Theta+1-1 / \Theta}}\left(1+\Theta \ln \Theta\left[\frac{\Theta+1+2 \Gamma}{(\Theta-1)^{2}}\right]\right) \\
& =25.8 \delta_{L} \approx 1.5 \times 10^{-3} \mathrm{~m}
\end{aligned}
$$

where $\Theta=\rho_{u} / \rho_{b} \approx 3$ is the ratio of the unburnt gas density $\rho_{u}$ to the burnt gas density $\rho_{b}$, and the factor $\Gamma$ only depends on $\Theta$ :

$$
\Gamma(\Theta)=\frac{\Theta}{\Theta+1}\left(\sqrt{\Theta+1-\frac{1}{\Theta}}-1\right) \approx 0.686 .
$$

During the linear stage of DL instability the amplitude of the perturbations grows exponentially, $A(z, t) \sim \exp (\sigma t+\mathrm{i}(2 \pi / \lambda) z)$, where $\sigma$ is the increment of the instability growth, $t$ is time, $\lambda$ the perturbation wavelength and $z$ the coordinate in the spanwise direction. The fastest growing perturbations are characterized by the wavelength

$$
\lambda=2 \lambda_{c} \approx 51.6 \delta_{L} \approx 3 \times 10^{-3} \mathrm{~m},
$$

resulting in approximately five fastest growing perturbation wavelengths in the domain's spanwise direction, compare with figure $3(b, c)$. Thus, the nearly periodic structure of the flame front observed in figure $3(b, c)$ may be attributed to the fact that the fastest growing perturbations with wavelengths approximately equal to $1 / 5$ of the channel width may dominate over other harmonics as the DL instability develops. However, at later times in the simulation the periodic structure is modified due to the influence of the incoming flow. The increment of growth for the fastest growing harmonics can be estimated as in Bychkov \& Liberman (2000)

$$
\sigma_{D L}=\Gamma(\Theta) S_{l} k\left(1-k / k_{c}\right)=\Gamma(\Theta) S_{l} \frac{\pi}{2 \lambda_{c}} \approx 9.9 \times 10^{3} \mathrm{~s}^{-1},
$$

where $k$ is the perturbation wavenumber and $k_{c}=(2 \pi) / \lambda_{c}$ is the wavenumber corresponding to $\lambda_{c}$. The resulting time scale of the DL instability development is $\tau_{D L}=1 / \sigma_{D L} \approx 1.0 \times 10^{-4} \mathrm{~s}$ which is compatible with the observed temporal evolution of the initial instability of the flame sheet, see figure $3(b)$.

In order to obtain an estimate for a relevant time scale that characterizes the approaching turbulence, we use the size of the boundary layer streaks in the 
longitudinal direction and the convective velocity associated with them. The convective velocity of the boundary layer streaks is estimated by Kim \& Hussain (1994) to be $u_{c} \approx 0.55 \cdot U_{c}^{f} \approx 14 \mathrm{~m} \mathrm{~s}^{-1}$, where $U_{c}^{f}$ is the channel centreline mean streamwise velocity. Furthermore, the size of the boundary layer streaks in the streamwise direction is approximately 300 wall units that correspond, in the present case, to a length scale $\lambda_{I x} \approx 8.0 \times 10^{-3} \mathrm{~m}$. The eddy passage time can therefore be estimated as $\lambda_{I x} / u_{c} \approx 5.0 \times 10^{-4} \mathrm{~s}$ which is similar to the time span of the simulation. The size and spacing of the boundary layer streaks in the spanwise direction are approximately 30 and 100 wall units (Kim \& Moin 1989; Jiménez 1998), resulting in an estimated perturbation wavelength, $\lambda_{I z} \approx 2.5 \times 10^{-3} \mathrm{~m}$ or $\sim 1 / 6$ of the spanwise domain width. This spatial scaling is of the same order of magnitude as the size of fastest growing perturbations already estimated in (3.3), thus justifying time scale separation (Chaudhuri et al. 2011).

Summarizing, the relevant turbulent time and frequency scales are

$$
\tau_{\text {turb }}=\frac{\lambda_{\text {Ix }}}{u_{c}} \approx 5.0 \times 10^{-4} \mathrm{~s}, \quad \omega_{\text {turb }}=1 / \tau_{\text {turb }} \approx 2.0 \times 10^{3} \mathrm{~s}^{-1} .
$$

Since $\sigma_{D L} \gtrsim \omega_{t u r b}$ (see (3.4) and (3.5)), the DL hydrodynamic instability is expected to be sustained in the present turbulent flow (Chaudhuri et al. 2011) and the flame propagates as a wrinkled flamelet or corrugated flamelet. Owing to the anisotropy and inhomogeneity of the approaching turbulent boundary layer flow the analytical estimation of the turbulence-induced DL cut-off wavelength and, consequently, the turbulent flame speed (Chaudhuri et al. 2011) is hindered. However, the lower-bound analytical estimate of the contribution of the DL instability to the turbulent flame speed can be obtained using the following expression for the maximum curved flame velocity as a first step of the DL instability cascade (Bychkov \& Liberman 2000)

$$
S_{D L}=S_{l}\left(1+\frac{\Theta(\Theta-1)^{2}}{\Theta^{3}+\Theta^{2}+3 \Theta-1}\right) \approx 1.3 S_{l} .
$$

This gives a smaller value compared with the maximum value of the turbulent flame speed obtained from the numerical results (see the next section) and suggests a non-negligible contribution to the turbulent flame speed by boundary layer turbulence. It is noted that the velocity amplification by the DL hydrodynamic instability is limited in amplitude due to spanwise confinement in the present case (Bychkov \& Liberman 2000), even though it is quite fast developing at its initial stage because of the large value of $S_{l}$.

\subsection{Flashback speed estimates}

In this section the upstream propagation velocity of the turbulent flame (flashback speed) in the channel is quantified. The propagation velocity is determined as follows.

(a) For each of the available samples, the most upstream position of the flame leading edge (bulges) in the streamwise direction is identified.

(b) The displacement of the most upstream position of the flame is plotted as a function of time.

The flame propagation speed thus obtained is shown in figure 10 for TCF01 and TCF02 indicating, in general, relatively high flashback speeds for the simulated flames. Note that the flame propagation velocities at 1 and $2 \mathrm{~atm}$ are normalized by their respective laminar values and that the flashback speed is larger at the higher 
(a)

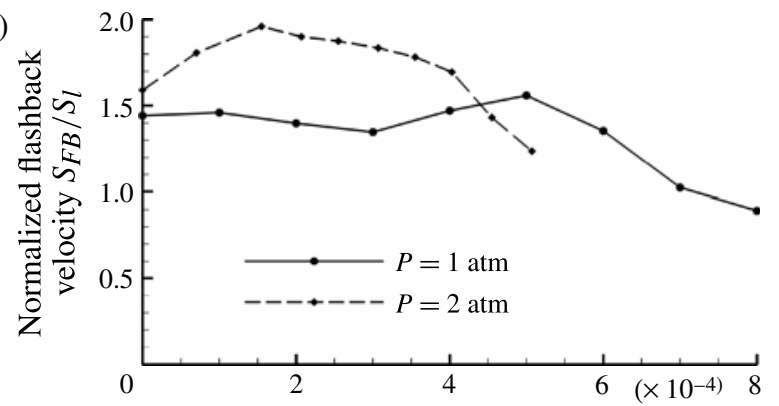

(b)

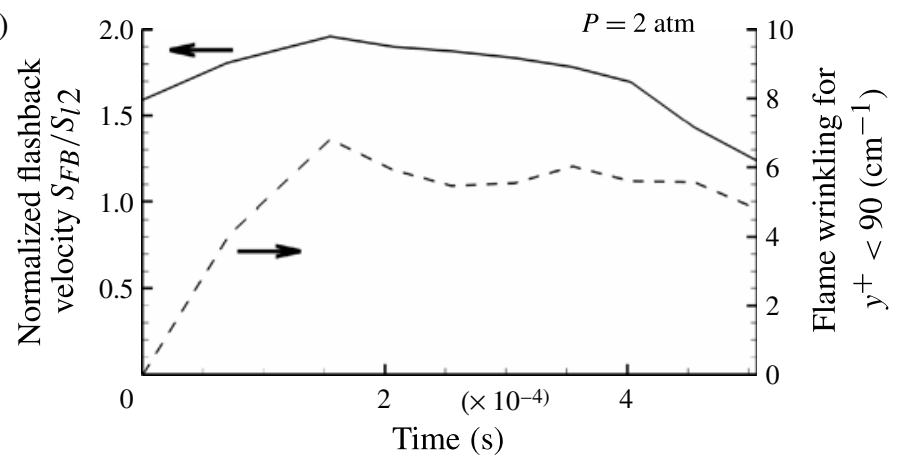

FIGURE 10. (a) Flashback speed estimates at 1 and 2 atm shown as a function of simulation time. (b) Temporal evolution of flashback speed and flame wrinkling at 2 atm.

pressure, see figure $10(a)$. The temporal evolution of the flame propagation velocity is compared with that of a flame wrinkling index in figure $10(b)$ for TCF02. The flame wrinkling index is extracted as the scalar integral, computed on the $C=0.7$ surface and conditional for $y^{+}<90$, on the absolute value of the surface curvature.

The same pattern is observed in the temporal evolution of the flashback speed for both case TCF01 and case TCF02: first the flame accelerates, then, after reaching a maximum velocity, the flame propagation speed stabilizes around a constant value until a marked speed reduction takes place towards the end of the simulation. The observed behaviour can be explained as follows.

(a) The initially flat flame accelerates after being wrinkled by the combined effect of the approaching turbulence and of the DL hydrodynamic instability.

(b) Once the maximum flame wrinkling induced by the interaction of the approaching turbulence with the flame sheets is reached and the effect of the DL instability becomes negligible, the flashback velocity fluctuates around a mean value.

(c) As the flame sheet approaches the upstream inlet, the imposed boundary conditions on the streamwise velocity component weaken the backflow regions induced by the flame, reducing their physical extent (figure $3 g$ ) and ultimately leading to their disappearance (figure $3 h$ ), thereby negatively affecting the flashback speed.

(d) The reduction in the flashback speed, due initially to the weakening and subsequently to the disappearance of the backflow regions, occurs upstream and independently of the reduction in flame wrinkling caused by the interaction of the flame with the upstream inlet boundary. 
(e) At the very end of the simulation, flame wrinkling is also affected by the inlet boundary conditions that artificially flatten the most upstream flame portions (bulges), see the red surface in figure $3(h)$ and the dashed line in figure $10(b)$.

The last three statements above require further consideration. Although the imposed velocity boundary conditions artificially affect the behaviour of the physical phenomenon that is being studied, the observed interaction of the boundary conditions with the backflow regions of the flow provide useful insight. In fact, the effect of the upstream boundary conditions on reducing the regions of flow reversal, i.e. causing a reduction in flashback speed and in flame wrinkling at late times, also demonstrates that these backflow regions are responsible for enabling and sustaining the high flashback speeds observed here.

The observed pressure scaling for the flashback speed is also interesting and gives higher flame velocities at higher pressure for the present configuration. The thickness of the flame front is reduced at higher pressure while the density jump remains nearly unchanged. Therefore, the scalar gradients increase across the flame front that, in turn, enhance the hydrodynamic DL instability leading to a more wrinkled flame surface and to a faster acceleration of the flame. This is shown for the case of freely propagating flames expanding in quiescent fluid by Law, Jomaas \& Bechtold (2005), that is, independent of the time and length scales of the approaching turbulence. It is also observed for burner stabilized turbulent flames by Kobayashi \& Kawazoe (2000). Furthermore, the present results suggest a ratio of turbulent flashback velocities, $1.9 / 1.5=1.29$, for pressure of $1-2 \mathrm{~atm}$ while the correlation proposed in the paper by Kobayashi \& Kawazoe (2000) yields a ratio of 1.32 for the same conditions, indicating a surprisingly good pressure scaling agreement between turbulent boundary layer flashback flames and burner stabilized turbulent flames.

\subsection{Flame heat release rate}

The heat release rate from the premixed flame changes considerably depending on the position of the flame itself, and of notable importance is its distance from the wall. This topic was discussed in Gruber et al. (2010) for the case of an anchored flame whose flame brush impinges onto the wall. Regions of the flame that do not feel the presence of the wall behave well in accordance to established knowledge about fuel-rich, freely-propagating, premixed flames. Most importantly, as already observed in Gruber et al. (2010), the heat release rate from the flame increases by an order of magnitude at quenching during the FWI due to exothermic, zero-activation energy, radical recombination reactions taking place at the 'cold' isothermal wall. This observation is confirmed in the present results. Close inspection of the near-wall region of the flame surface reveals the steep increase in heat release rate at the wall (blackcoloured region in figure 11a). The same characteristic behaviour can also be observed in the scatter plot of figure 12 that shows the heat release rate as a function of the reaction progress variable $C$ for several wall distances, where the purple-coloured branch of the scatter points represents the heat release rate at the wall.

More details about the quenching process that takes place during the FWI can be observed in figure 11(b) that illustrates the behaviour of the hydrogen reaction rate. The hydrogen fuel consumption stops at $y^{+} \sim 2$ above the wall where the flame is quenched, however radical recombination reactions provide for production of hydrogen at the wall directly below the quenching point (white-coloured region near $x^{+} \sim 100$ in figure $11 b$ ). As soon as it is formed by the radical recombination reactions, the light hydrogen molecule escapes away from the wall region towards the hotter products 
(a)

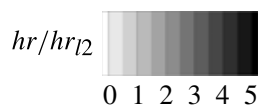

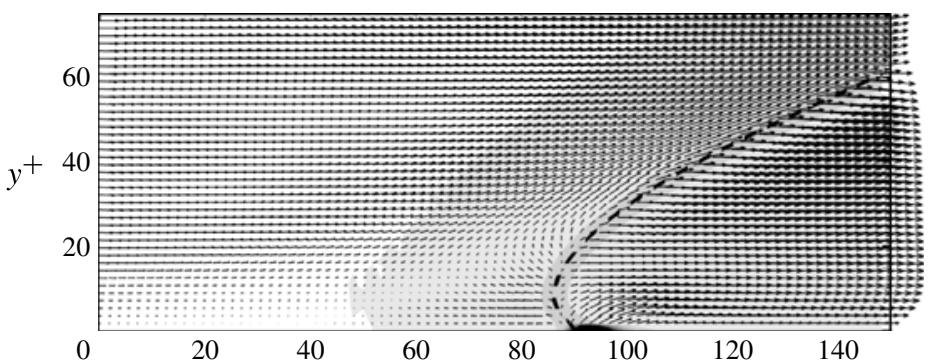

(b) $R R H 2\left(\mathrm{~s}^{-1}\right)$
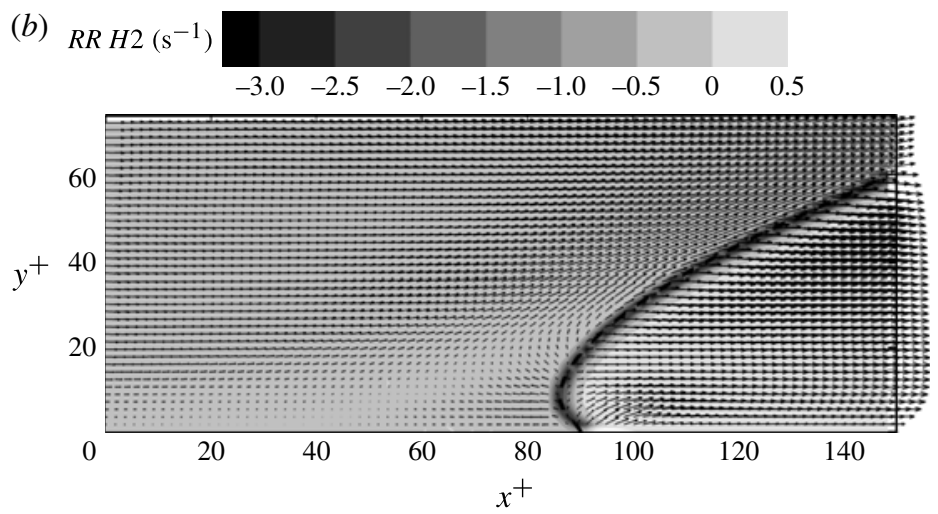

FIGURE 11. Details of the heat release rate $(a)$ and of the hydrogen reaction rate $(b)$ are given on $x y$-planes oriented normal to both the wall and the flame bulge at its most upstream position (point ' $\mathrm{O}$ ' in the idealization of figure 4). The flame is denoted by the reaction progress variable $C=0.7$ in both figures (black dashed line), where unburnt mixture is on the left and burnt mixture is on the right of the lines.

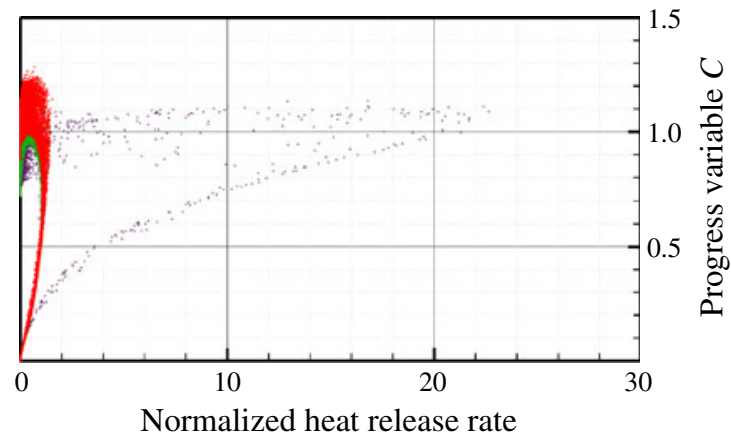

FIGURE 12. Scatter plot of the instantaneous heat release rate as a function of reaction progress variable $C$ and of wall distance: purple-coloured points, $y^{+}<5$; green-coloured points, $5<y^{+}<30$; and red-coloured points, $y^{+}>30$.

stream due to the large temperature gradient at the isothermal wall. However, because of the deficiency of oxygen in the products stream, the fuel arriving there cannot 
burn, at least in the present configuration. It is reasonable to assume that, in the case of fuel lean mixtures, the hydrogen generated at the wall by radical recombination reactions would then diffuse into the product stream containing oxygen and therefore burn behind the main flame front.

\section{Conclusions}

Three-dimensional DNSs of upstream flame propagation in turbulent plane channel flow have been performed for premixed $\mathrm{H}_{2}$-air flames at pressures of 1 and 2 atm. The present DNS study is a modification of a previous one (Gruber et al. 2010) obtained by removing the flame anchor, thereby allowing the premixed flames to freely propagate upstream against the bulk flow of the reactants (at $R e_{\tau}=180$ ). The stoichiometry and the temperature of the $\mathrm{H}_{2}$-air mixtures are left unchanged compared with the earlier case of $\phi=1.5$ and $T_{u}=750 \mathrm{~K}$, respectively. The aim of the present DNS study is to investigate the mechanism of premixed flame flashback in turbulent boundary layers.

Analysis of transient flame propagation in the turbulent boundary layers of the channel reveals the presence of near-wall $\left(y^{+}<20\right)$ regions of reverse flow immediately ahead of the upstream-propagating flame portions that are convex towards the reactants. While these particular features of boundary layer premixed flame flashback have never been previously observed in modelling studies, their presence is confirmed by some recent findings from a parallel experimental effort (Eichler \& Sattelmayer 2012) conducted within BIGCO2/BIGCCS. The present results show that the widely used boundary layer flashback model (Lewis \& von Elbe 1943), based on the assumption that the flame has negligible effect on the approaching reactant flow, is largely inadequate. Moreover, the presence of near-wall regions of reverse flow ahead of the flame sheet has implications on flashback speed: it actively contributes to flame acceleration (together with wrinkling due to DL instability and turbulence) and it is instrumental in maintaining high flame propagation velocities. The observed pressure scaling for the flashback speed indicates higher flame velocity at higher pressure and the velocity ratio obtained for the present configurations is in reasonable agreement with a correlation found in the literature for burner stabilized flames (Kobayashi \& Kawazoe 2000). Finally, correct representation of the tiny near-wall reverse flow patterns, and thereby of wall flashback, poses strict near-wall resolution requirements for LES models and a considerable modelling issue for hybrid LES-RANS (Reynoldsaveraged Navier-Stokes) models that employ simplified descriptions of the boundary layer.

\section{Acknowledgements}

The work at SINTEF has been supported by the BIGCCS Centre, performed under the Norwegian research program Centres for Energy Efficient Research. The authors acknowledge the following partners for their contributions: Aker Solutions, ConocoPhilips Skandinavia AS, Det Norske Veritas AS, Gassco AS, Hydro Aluminium AS, Shell Technology AS, Statkraft Development AS, StatoilHydro Petroleum AS, TOTAL E\&P Norge AS and the Research Council of Norway (grant numbers 178004/I30 and 176059/I30). Computational support for this project was provided by, and this research used the resources of, the National Center for Computational Sciences at Oak Ridge National Laboratory, which is supported by the office of Science of the US Department of Energy under contract DE-AC0500OR22725. The work at Sandia National Laboratories was supported by the Division 
of Chemical Sciences, Geosciences, and Biosciences, Office of Basic Energy Sciences of the US Department of Energy and by the Combustion Energy Frontier Research Center. SNL is a multiprogramme laboratory operated by Sandia Corporation, a Lockheed Martin Company for the US DOE under Contract DE-AC04-94AL85000. The work at Princeton University was also supported by the Combustion Energy Frontier Research Center of the US Department of Energy.

\section{REFERENCES}

AlshaAlan, T. M. \& Rutland, C. J. 1998 Turbulence, scalar transport, and reaction rates in flame-wall interaction. In Twenty-Seventh Symposium (International) on Combustion, pp. 793-799. The Combustion Institute.

Alshaflan, T. M. \& Rutland, C. J. 2002 Wall heat flux in turbulent premixed reacting flow. Combust. Sci. Technol. 174, 135-165.

BLINT, R. J. 1986 The relationship of the laminar flame width to flame speed. Combust. Sci. Technol. 49, 79-92.

Bolland, O. \& Undrum, H. 2003 A novel methodology for comparing $\mathrm{CO}_{2}$ capture options for natural gas-fired combined cycle plants. Adv. Environ. Res. 7 (4), 901-911.

Bruneaux, G., Akselvoll, K., Poinsot, T. \& Ferziger, J. H. 1996 Flame-wall interaction simulation in a turbulent channel flow. Combust. Flame 107, 27-44.

Bychkov, V. V. \& Liberman, M. A. 2000 Dynamics and stability of premixed flames. Phys. Rep. 325, 115-237.

CARRoni, R. 2006 Development of a gas turbine burner for the lean-premixed combustion of $\mathrm{H}_{2}$-rich fuels. In 8th International Conference on Greenhouse Gas Control Technologies, Trondheim, Norway, 19th-22nd June 2006. IEAGHG.

Chaudhuri, S., Akkerman, V. \& Law, C. K. 2011 Spectral formulation of turbulent flame speed with consideration of hydrodynamic instability. Phys. Rev. E 84, 026322.

Chen, J. H., Choudhary, A., De Supinski, B., DeVries, M., Hawkes, E. R., Klasky, S., Liao, W. K., Ma, K. L., Mellor-Crummey, J., Podhorski, N., Sankaran, R., Shende, S. \& Yoo, C. S. 2009 Terascale direct numerical simulations of turbulent combustion using S3D. Comput. Sci. Disc. 2, 1-31.

Chiesa, P., Lozza, G. \& MAzzocchi, L. 2005 Using hydrogen as gas turbine fuel. ASME J. Engng Gas Turbines Power 127, 73-80.

Creta, F., Fogla, N. \& Matalon, M. 2011 Turbulent propagation of premixed flames in the presence of Darrieus-Landau instability. Combust. Theor. Model. 15 (2), 267-298.

Dabireau, F., Cuenot, B., Vermorel, O. \& Poinsot, T. 2003 Interaction of flames of $\mathrm{H}_{2}+\mathrm{O}_{2}$ with inert walls. Combust. Flame 135, 123-133.

ECHEKKI, T. \& Chen, J. H. 2003 Direct numerical simulation of autoignition in non-homogeneous hydrogen-air mixtures. Combust. Flame 134, 169-191.

Egolfopoulos, F. N., ZhANG, H. \& ZHANG, Z. 1997 Wall effects on the propagation and extinction of steady, strained, laminar premixed flames. Combust. Flame 109, $237-252$.

Eichler, C., Baumgartner, G. \& Sattelmayer, T. 2011 Experimental investigation of turbulent boundary layer flashback limits for premixed hydrogen-air flames confined in ducts. In Proceedings of ASME Turbo Expo 2011, June 6-10, 2011, Vancouver, Canada, pp. GT2011-45362. American Society of Mechanical Engineers.

Eichler, C. \& SATtelmayer, T. 2011 Experiments on flame flashback in a quasi-2D turbulent wall boundary layer for premixed methane-hydrogen-air mixtures. ASME J. Engng Gas Turbines Power 133, 011503.

EICHLER, C. \& SATtelmayeR, T. 2012 Premixed flame flashback in wall boundary layers studied by long-distance micro-PIV. Exp. Fluids 52 (2), 347-360.

Ezekoye, O., GReif, R. \& SAWYeR, R. F. 1992 Increased surface temperature effects on wall heat transfer during unsteady flame quenching. In Proceedings 24th International Symposium on Combustion, pp. 1465-1472. The Combustion Institute.

Fritz, J., KRÖNer, M. \& SATtelmayer, T. 2004 Flashback in a swirl burner with cylindrical premixing zone. ASME J. Engng Gas Turbines Power 126, 276-283. 
Grout, R. W., Gruber, A., Kolla, H., Bremer, P.-T., Bennet, J. C., Gyulassy, A. \& CHEN, J. H. 2012 A direct numerical simulation study of turbulence and flame structure in transverse jets analysed in jet-trajectory based coordinates. J. Fluid Mech. 706, 351-383.

Grout, R. W., Gruber, A., Yoo, C. S. \& Chen, J. H. 2011 Direct numerical simulation of flame stabilization downstream of a transverse fuel jet in cross-flow. Proc. Combust. Inst. 33 (1), 1629-1637.

Gruber, A., Sankaran, R., Hawkes, E. R. \& Chen, J. H. 2010 Turbulent flame-wall interaction: a direct numerical simulation study. J. Fluid Mech. 658, 5-32.

HAWKES, E. R. \& CHEN, J. H. 2005 Evaluation of models for flame stretch due to curvature in the thin reaction zones regime. In Proceedings 30th International Symposium on Combustion, pp. 647-655. The Combustion Institute.

Hawkes, E. R., Sankaran, R., Sutherland, J. C. \& Chen, J. H. 2007 Scalar mixing in direct numerical simulations of temporally evolving plane jet flames with skeletal co/h2 kinetics. In Proceedings 31th International Symposium on Combustion, pp. 1633-1640. The Combustion Institute.

Heeger, C., Gordon, R. L., Tummers, M. J., SAttelmayer, T. \& Dreizler, A. 2010 Experimental analysis of flashback in lean premixed swirling flames: upstream flame propagation. Exp. Fluids 49, 853-863.

Hocks, W., Peters, N. \& Adomeit, G. 1981 Flame quenching in front of a cold wall under two-step kinetics. Combust. Flame 41, 157-170.

JiméneZ, J. 1998 The largest scales of turbulent wall flows. In Annual Research Briefs of the Center for Turbulence Research, Stanford University, CA, pp. 137-154.

Kee, R. J., Dixon-lewis, G., Warnatz, J., Coltrin, M. E., Miller, J. A. \& Moffat, H. K. 1999 A fortran chemical kinetics package for the analysis of gas-phase chemical kinetics. Tech. Rep. Release 3.5. Reaction Design Inc., San Diego, CA.

Kennedy, C. A. \& CARpenter, M. H. 1994 Several new numerical methods for compressible shear-layer simulations. Appl. Numer. Maths 14 (0), 397-433.

Kennedy, C. A., CARpenter, M. H. \& Lewis, R. M. 2000 Low-storage, explicit Runge-Kutta schemes for the compressible Navier-Stokes equations. Appl. Numer. Maths 35 (0), 177-219.

KIM, J. \& HUSSAIN, F. 1994 Propagation velocity of perturbations in turbulent channel flow. Phys. Fluids 5, 695-706.

KIM, J. \& MoIN, P. 1989 Transport of passive scalars in a turbulent channel flow. In Proceedings 6th International Symposium on Turbulent Shear Flow, 7-9 September, Tolouse, France, pp. 85-96.

Kobayashi, H. \& KaWAzOE, H. 2000 Flame instability effects on the smallest wrinkling scale and burning velocity of high-pressure turbulent premixed flames. Proc. Combust. Inst. 28 (1), 375-382.

Kolla, H., Grout, R. W., Gruber, A. \& Chen, J. H. 2012 Mechanisms of flame stabilization and blowout in a reacting turbulent hydrogen jet in cross-flow. Combust. Flame 159 (8), 2755-2766.

Kurdyumov, V. N. \& Fernandez-TARrazo, E. 2002 Lewis number effect on the propagation of premixed laminar flames in narrow open ducts. Combust. Flame 128, 382-394.

Kurdyumov, V. N., Fernandez, E. \& Linan, A. 2000 Flame flashback and propagation of premixed flames near a wall. Proc. Combust. Inst. 28 (1), 1883-1889.

Kurdyumov, V. N., Fernandez-Tarrazo, E., Truffaut, J. M., Quinard, J., Wangher, A. \& SeArby, G. 2007 Experimental and numerical study of premixed flame flashback. Proc. Combust. Inst. 31 (1), 1275-1282.

LAW, C. K. 2006 Combustion Physics, 1st edn. Cambridge University Press.

LAw, C. K., JomaAs, G. \& BEChtold, J. K. 2005 Cellular instabilities of expanding hydrogen/propane spherical flames at elevated pressures: theory and experiment. Proc. Combust. Inst. 30 (1), 159-167.

LEE, S. T. \& T'IEN, J. S. 1982 A numerical analysis of flame flashback in a premixed laminar system. Combust. Flame 48, 273-285.

Lewis, B. \& VON Elbe, G. 1943 Stability and structure of burner flames. J. Chem. Phys. 11, 75-97.

Li, J., Zhao, Z., Kazarov, A. \& Dryer, F. L. 2004 An updated comprehensive kinetic model of hydrogen combustion. Intl J. Chem. Kinet. 36, 566-575. 
Lipatnikov, A. N. \& Chомiak, J. 2010 Effects of premixed flames on turbulence and turbulent scalar transport. Prog. Energy Combust. Sci. 36, 1-102.

Mayer, C., Sangl, J., Sattelmayer, T., Lachaux, T. \& Bernero, S. 2011 Study on operational window of a swirl stabilized syngas burner under atmospheric and high pressure conditions. In Proceedings of ASME Turbo Expo 2011, June 6-10, 2011, Vancouver, Canada, pp. GT2011-45125. American Society of Mechanical Engineers.

Moser, R., KIM, J. \& MANSOUR, N. 1999 Direct numerical simulation of turbulent channel flow up to $R e_{\tau}=590$. Phys. Fluids 11 (4), 943-945.

Poinsot, T., Haworth, D. C. \& Bruneaux, G. 1993 Direct simulation and modeling of flame-wall interaction for premixed turbulent combustion. Combust. Flame 95, 118-132.

Poinsot, T. \& LELE, S. K. 1992 Boundary conditions for direct simulations of compressible viscous flow. J. Comput. Phys. 101, 104-129.

Poinsot, T. \& Veynante, D. 2001 Theoretical and Numerical Combustion, 1st edn. Edwards.

PopP, P. \& BAUM, M. 1997 Analysis of wall heat fluxes, reaction mechanisms, and unburnt hydrocarbons during the head-on quenching of a laminar methane flame. Combust. Flame 108, 327-348.

Popp, P., Smooke, M. \& BAUM, M. 1996 Heterogeneous/homogeneous reaction and transport coupling during flame-wall interaction. In Proceedings 26th International Symposium on Combustion, pp. 2693-2700. The Combustion Institute.

Sankaran, R., Hawkes, E. R., Chen, J. H., Lu, T. \& Law, C. K. 2007 Structure of a spatially developing turbulent lean methane-air bunsen flame. In Proceedings 31th International Symposium on Combustion, pp. 1291-1298. The Combustion Institute.

SANkARAN, R., IM, H. G., HAWKes, E. R. \& CHEN, J. H. 2005 The effects of non-uniform temperature distribution on the ignition of a lean homogeneous hydrogen-air mixture. In Proceedings 30th International Symposium on Combustion, pp. 875-882. The Combustion Institute.

Sutherland, J. C. \& Kennedy, C. A. 2003 Improved boundary conditions for viscous, reactive, compressible flows. J. Comput. Phys. 191, 502-524.

Westbrook, C. K., Adamczyk, A. A. \& Lavoie, G. A. 1981 A numerical study of laminar flame wall quenching. Combust. Flame 40, 81-99.

Williams, F. A. 1985 Combustion Theory, 2nd edn. Addison Wesley Publishing Company.

Yoo, C. S. \& IM, H. G. 2007 Characteristic boundary conditions for simulations of compressible reacting flows with multi-dimensional, viscous and reaction effects. Combust. Theor. Model. 11 (2), 259-286.

Yoo, C. S., SAnkaran, R. \& Chen, J. H. 2009 Three-dimensional direct numerical simulation of a turbulent lifted hydrogen jet flame in heated coflow: flame stabilization and structure. J. Fluid Mech. 640, 453-481.

Yoo, C. S., Wang, Y., Trouvé, A. \& IM, H. G. 2005 Characteristic boundary conditions for direct simulations of turbulent counterflow flames. Combust. Theor. Model. 9 (4), 617-646. 